\title{
Imaging the wavefunctions of a quantum dot system
}

\author{
O. Di Stefano*, S. Savasta, G. Martino, and R. Girlanda \\ INFM and Dipartimento di Fisica della Materia e Tecnologie Fisiche Avanzate, \\ Università di Messina Salita Sperone 31, 98166 Messina, Italy
}

\begin{abstract}
We describe how, exploiting the current scanning near-field optical techniques, it is possible to map phase and modulus of the wavefunctions of a quantum dot system. In particular we propose an experimental setup based on an interferometric technique able to measure simultaneously modulus and phase of wavefunctions. We present numerical calculations simulating the measurements on dots arising from interface fluctuations of GaAs quantum wells.
\end{abstract}

Recent measurements based on spatially-resolved photoluminescence provided direct information on the spatial and energy distribution of light emitting nanometric centers [1-5] of semiconductor quantum structures. However spatially resolved photoluminescence provides only an indirect probe of the electronic structure. Recently Guest et al. [6] presented a technique that combines coherent nonlinear spectroscopy with near-field microscopy providing a means to map the local optical density of states (LDOS) of the system in analogy to scanning tunneling microscopy measurements $[7,8]$ that mapped out the electronic LDOS. Coherent optical control and optical manipulation play a fundamental role in the functioning of proposed devices. Proposed device architectures ultimately rely on tuning the interactions between individual electronic states, that requires direct access to eigenstates with spectral and spatial selectivity [9].

Here we show that it is possible to go beyond LDOS measurements, thus achieving the ultimate characterization tool for a mesoscopic quantum system. We propose a near-field optical technique able to map modulus and phase of the wavefunctions of a quantum dot system. Recently Phillips et al. [10] advanced from the more traditional use of the scanning near-field optical microscope (SNOM) for electromagnetic imaging measuring both the light intensity and phase in the near field. We show that, when the probed region of the sample is comparable or lower than the spatial variations of the excitonic wavefunction, the phase and modulus of the collected light can be related to the phase and modulus of the excitonic wavefunction. In order to simulate the measurements, we consider a system of quantum dots arising from interface fluctuations of GaAs quantum wells. The numerical calculations consist of two steps in close analogy with real experiments. The first step is the growth of the sample corresponding to a single realization of disorder with given statistical properties. Once the sample has been obtained, we calculate the SNOM signal acquired in collection mode. In collectionmode SNOM, the sample is usually illuminated by a laser focussed onto the sample surface. An optical fiber (waveguide) with a sharp tip is scanned along the sample at subwavelength distance (in the near-field region), and the signal delivered by an optical detector at the end of the fiber is recordered. In experiments done in collection mode the relevant optical field that can be detected by a

\footnotetext{
* Corresponding author: e-mail: distef@ortica.unime.it
} 
general near-field setup is given by [11] $S^{\text {tot }}(\omega)=A^{b g}(\omega)+S_{g}(\omega)$, where $A^{b g}(\omega)$ is the background signal assumed to be uniform along the $x-y$ plane and

$$
S_{g}(\omega)=A(\omega) \int \mathrm{d} z \mathrm{~d} \boldsymbol{r} \boldsymbol{P}(\boldsymbol{r}, z, \omega) \cdot \boldsymbol{E}_{\text {out }}(\boldsymbol{r}, z, \omega),
$$

$A$ is a complex constant depending on the impedance of the material constituting the tip [11], $\boldsymbol{P}(\boldsymbol{r}, z, \omega)$, is the polarization density of the sample induced by an electromagnetic field $\boldsymbol{E}_{\text {in }}(\boldsymbol{r}, z, \omega)$. $\boldsymbol{E}_{\text {out }}(\boldsymbol{r}, z, \omega)$ is the signal mode delivered by a collection mode SNOM. We have indicated with $\boldsymbol{r} \equiv(x, y)$ the projection of the position vector on the plane of the QW and with $z$ the coordinate along the growth direction. Following the general linear response theory [12], the linear macroscopic polarization $\boldsymbol{P}(\boldsymbol{r}, z, \omega)$ can be written as

$$
\boldsymbol{P}(\boldsymbol{r}, z, \omega)=\int \overrightarrow{\boldsymbol{\chi}}\left(\boldsymbol{r}, z ; \boldsymbol{r}^{\prime}, z ; \omega\right) \cdot \boldsymbol{E}_{\text {in }}\left(\boldsymbol{r}^{\prime}, z^{\prime}, \omega\right) \mathrm{d} \boldsymbol{r}^{\prime} \mathrm{d} z^{\prime},
$$

where $\overrightarrow{\boldsymbol{\chi}}\left(\boldsymbol{r}, z ; \boldsymbol{r}^{\prime}, z ; \omega\right)$ is the nonlocal susceptibility tensor. In systems with extended wave-functions such as semiconductors nonlocality plays a relevant role [13, 14]. In reasonable good quality QWs the amplitude of the confinement energy fluctuations are typically one order of magnitude smaller than the binding energy of the $1 S$ exciton [15]. In this limit, the susceptibility tensor is given by [13, 14] $\overrightarrow{\boldsymbol{\chi}}\left(\boldsymbol{r}, \boldsymbol{r}^{\prime}, z, z^{\prime}\right)=\vec{\chi}\left(\boldsymbol{r}, \boldsymbol{r}^{\prime}\right) \rho(z) \rho\left(z^{\prime}\right)$, where $\rho(z)$ is the product of the electron and hole envelope functions along the growth axis and

$$
\overleftrightarrow{\chi}\left(\boldsymbol{r}, \boldsymbol{r}^{\prime}\right)=-\mu^{2}\left|\phi_{1 s}(0)\right|^{2} \sum_{n} \frac{\psi_{n}(\boldsymbol{r}) \psi_{n}^{*}\left(\boldsymbol{r}^{\prime}\right)}{\omega+i \gamma-E_{n}} \overleftrightarrow{\mathbf{1}},
$$

where $\psi_{n}$ are mesoscopic wavefunctions describing the excitonic centre of mass motion.

Inserting Eq. (2) into Eq. (1) and observing that in the collection mode the input field can be considered constant along the sample, we obtain $S=A \int \mathrm{d} \boldsymbol{r} \mathrm{d} \boldsymbol{r}^{\prime} \chi\left(\boldsymbol{r}, \boldsymbol{r}^{\prime}, \omega\right) \boldsymbol{E}_{\text {in }} \cdot \boldsymbol{E}_{\text {out }}\left(\boldsymbol{r}^{\prime}, \omega\right)$, where $\boldsymbol{E}_{\text {in/out }}(\boldsymbol{r}, \omega)=\int \boldsymbol{E}_{\text {in/out }}(\boldsymbol{r}, z, \omega) \rho(z) \mathrm{d} z$. For the sake of simplicity, we describe the narrow light beam collected by the SNOM tip by a gaussian EM-field profile, $\boldsymbol{E}_{\text {in }} \cdot \boldsymbol{E}_{\text {out }}(\boldsymbol{r})=\mathcal{F}(\omega) g(\boldsymbol{r})$, where $g(\boldsymbol{r})=\exp \left[-\left(x^{2}+y^{2}\right) / \sigma^{2}\right]$. Using Eq. (3), the detected optical field (function of the frequency of input light and of the tip position $\boldsymbol{R})$ is thus given by

$$
S_{g}(\boldsymbol{R}, \omega)=\mathcal{A} \sum_{n} \frac{M_{n}^{*}}{\omega+i \gamma-E_{n}} \int \mathrm{d} \boldsymbol{r} g(\boldsymbol{r}-\boldsymbol{R}) \psi_{n}(\boldsymbol{r}),
$$

where $M_{n}$ is the exciton dipole moment and $\mathcal{A}$ includes all the constants obtained by the calculation. In the limit of very high spatial resolution, $\int \mathrm{d} \boldsymbol{r} g(\boldsymbol{r}-\boldsymbol{R}) \psi_{n}(\boldsymbol{r}) \rightarrow \psi_{n}(\boldsymbol{R})$. If in addition the homogeneous broadening $\gamma$ is much lower than the energy difference between the eigenstates of the system (as it usually happens in many quantum dot systems), the spatial variations of $S_{g}\left(\boldsymbol{R}, \omega=\omega_{n}\right)$ map $\psi_{n}(\boldsymbol{R})$. The phase and modulus of $S_{g}(\boldsymbol{R}, \omega)$ can be measured by an interferometric technique [10]. Unlike conventional SNOM, the optical signal from the probe does not pass directly to the detection electronics but is combined with a reference signal (a split fraction of the input laser beam with frequency $\omega$ and amplitude $L=l \mathrm{e}^{i \phi}$ ) as shown in Fig. 1. The two signals are combined by a MachZehnder interferometer operating in the balanced homodyne detection with a phase sensitive element

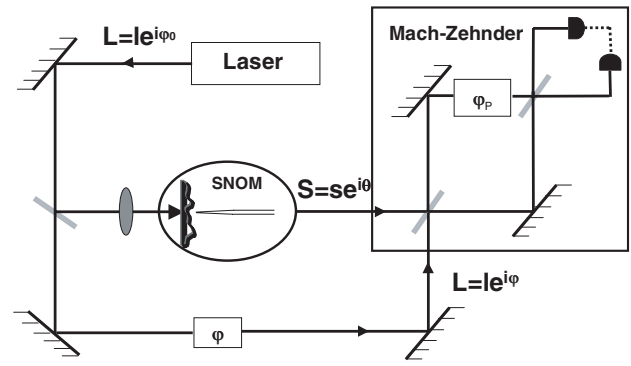

Fig. 1 Experimental setup able to detect both phase and amplitude of the system wavefunction. 
in the upper arm introducing a phase shift $\phi_{p}$. The output signal $W_{\text {out }}$ in the balanced homodyne detector is given by [16]: $W_{\text {out }}=\left[|L|^{2}-\left|S^{\text {tot }}\right|^{2}\right] \cos \left(\phi_{p}\right)+\left[L^{*} S^{\text {tot }}+S^{\text {tot* }} L\right] \sin \left(\phi_{p}\right)$. If $\phi_{p}=\pi / 2$, the signal detected is

$$
W_{\text {out }}=\left[L^{*} S^{\text {tot }}+S^{\text {tot* }} L\right]=W_{\text {out }, g}+W_{\text {out }}^{b g} .
$$

Observing that $A^{b g}=\left|A^{b g}\right| \mathrm{e}^{i \theta_{b g}}$ is the background signal and $S_{g}=\left|S_{g}\right| \mathrm{e}^{i \theta_{g}}$ is the SNOM signal (4) acquired in the collection mode measurement, we have $W_{\text {out }, g}=2 l\left|S_{g}\right| \cos \left(\theta_{g}-\phi\right)$ and $W_{\mathrm{out}}^{b g}=2 l\left|A^{b g}\right| \cos \left(\theta_{b g}-\phi\right)$. We observe from Eq. (5) that the second term in the r.h.s. gives (with fixed $\phi)$ a spatially uniform contribution and can be removed by different simple techniques. Hence the relevant signal is represented by the first term in the r.h.s. of Eq. (5). We also observe from Eq. (5) that the higher intensity of the output signal is obtained when $\phi=\theta_{g}$. Hence, tuning the laser frequency and varying the phase $\phi$ of the splitted laser beam, it is possible to map modulus and phase of the system wavefunctions. In the following we will present specific calculations for a system of quantum dots distributed on a two-dimensional surface [2,3]. We model the random potential $V(\boldsymbol{r})$ felt by the excitons as a zero mean, Gauss distributed and spatially correlated process. Calculations are carried out in real space mapping on a fine mesh of points the Hamiltonian [13] $H_{\boldsymbol{r}}=-\left(\hbar^{2} / 2 m\right) \nabla^{2}+V(\boldsymbol{r})$, that is then diagonalized in order to obtain eigenvalues and eigenvectors. We adopt an exciton kinetic mass of $m=0.25 m_{0}$ typical for AlAs/GaAs quantum wells. The spectra have been calculated by considering a square region of $0.48 \times 0.48 \mu \mathrm{m}^{2}$ which has been reproduced with a $60 \times 60$ mesh. Periodic boundary conditions have been adopted. For all the calculated spectra we used a homogeneous broadening $\gamma=20 \mu \mathrm{eV}$.

Figure $2 \mathrm{a}$ displays the 2D grey-scale image of the specific realization of the disorder potential used for the simulations. Before calculating the collected signal $S_{g}$, in order to get information on the local optical resonances of the system, we calculated absorption spectra under local illumination (FWHM = $25 \mathrm{~nm}$ ) [13]. Figure $2 \mathrm{~b}$ displays one of these spectra obtained with the beam centered at the position
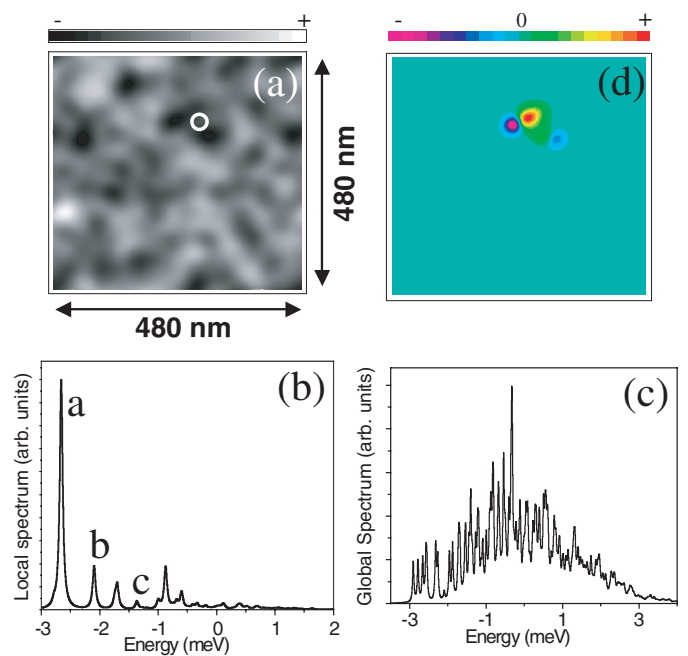

Fig. 2 (online colour at: www.interscience.wiley.com) a) specific realization of disorder potential in the region where local spectra have been calculated. The white circle specifies the location chosen to calculate the local spectrum shown in Fig. 2b; b) local spectrum; c) spectrum obtained using a beam with $\sigma=250 \mathrm{~nm}$ centered in the middle of the image in Fig. 3a; d) 2D image showing the wavefunction relative to the eigenvalue showed in Fig. $2 b$ by the letter b).

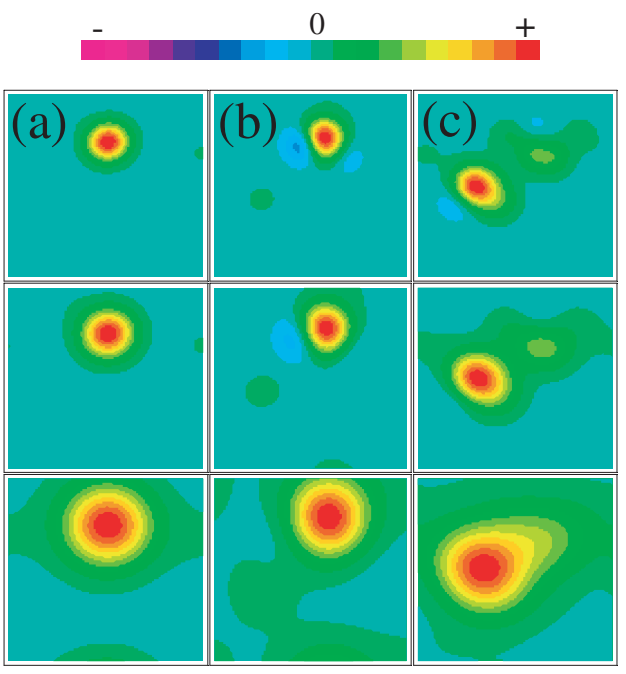

Fig. 3 (online colour at: www.interscience.wiley.com) a)-c) Images generated by sectioning the data $S_{g}(x, y, \omega)$ in planes of constant energy indicated by letters in Fig. 2b. The resolution is different for the three rows. The first row of images is obtained using a beam with a FWHM of $70 \mathrm{~nm}$. The second row of images is obtained using a beam with a FWHM of 94 $\mathrm{nm}$. The third row of images is obtained using a beam with a FWHM of $164 \mathrm{~nm}$. 
indicated by the white circle in Fig. 2a. The spectrum clearly shows sharp distinct peaks characteristic of zero-dimensional exciton states. We also report as reference the absorption spectrum obtained under uniform illumination of the sample. Figure $2 \mathrm{~d}$ displays the wavefunction $\psi_{b}(\boldsymbol{r})$ corresponding to the second excited state denoted by the letter b in Fig. $2 b$. Clearly sharp variations of the phase by $\pi$ (sign changes) can be noticed. We observe that, in the system under study, wavefunctions can be chosen as real and thus can display phase changes only by $\pi$. Figure 3 simulates what can be obtained by the set up shown in Fig. 1 for three different spatial resolutions. In particular it displays $\left|S_{g}\left(\omega_{n}\right)\right| \cos \theta_{g}\left(\omega_{n}\right)$ for three different values of the laser frequency $\omega$ corrosponding to the energy peaks labelled by letters in Fig. 2b. Each column displays results for a fixed energy and for different spatial resolution (from top to the bottom FWHM is respectively 70, 94 and $164 \mathrm{~nm}$ ). As expected, the fundamental state (column a) has a uniform phase, while the excited states display clear phase variations. We observe that the image displayed on column b) at FWHM $=70 \mathrm{~nm}$ reproduces well the modulus and phase variations of $\psi_{b}(\boldsymbol{r})$ (compare with Fig. 2d). The small differences between the two images are due to cancellation effects originating from the spatial interference of the corresponding quantum state due to the nonlocal character of light-matter interaction in semiconductors and to the finite resolution of the collecting tip [14, 13]. We also observe the dramatic impact of spatial resolution. Figure 3 shows that, lowering the resolution, information about the phase is lost more rapidly than information on the LDOS.

We have shown that it is possible to go beyond LDOS measurements, thus achieving the ultimate characterization tool for mesoscopic quantum systems. In particular we have proposed an experimental setup able to optically map simultaneously the phase and modulus of the wavefunctions of a quantum dot system. Numerical calculations on a GaAs quantum dot system displayed in Fig. 3 show that spatial resolutions available within current near-field techniques suffices. The method here proposed and analyzed can be applied to a large class of optically active two-dimensional mesoscopic quantum systems. Also of great interest would be the possible characterization of local phase changes when applying perturbations as magnetic and electric fields. Finally we believe that this technique would greatly assist the development of nano-devices based on tuning the interaction between individual electronic states.

\section{References}

[1] H. F. Hess, E. Betzig, T. D. Harris, L. N. Pfeiffer, and K. W. West, Science 264, 1740 (1994).

[2] D. Gammon, E. S. Snow, and D. S. Katzer, Appl. Phys. Lett. 67, 2391 (1995).

[3] D. Gammon, E. S. Snow, B. V. Shanabrook, D. S. Katzer, and D. Park, Phys. Rev. Lett. 76, 3005 (1996); Science 273, 87 (1996).

[4] Q. Wu, R. D. Grober, D. Gammon, and D. S. Katzer, Phys. Rev. Lett. 83, 2652 (1999).

[5] J. Hasen, L. N. Pfeiffer, A. Pinczuk, S. He, K. W. West, and B. S. Dennis, Nature (London) 390, 54 (1997).

[6] J. R. Guest, T. H. Stievater, Gang Chen, E. A. Tabak, B. G. Orr, D. G. Steel, D. Gammon, and D. S. Katzer, Science 293, 2224 (2001).

[7] R. Cingolani et al., Appl. Phys. Lett. 72, 148 (1998).

[8] S. G. Lemay et al., Nature 412, 617 (2001).

[9] A. Imamoglu et al., Phys. Rev. Lett. 83, 4204 (1999).

[10] P. L. Phillips, J. C. Knight, J. M. Pottage, G. Kakarantzas, and P. St. J. Russell, Appl. Phys. Lett. 76, 541 (2000).

[11] J-J. Greffet and R. Carminati, Prog. Surf. Sci. 56, 133 (1997).

[12] E. Runge and R. Zimmermann, phys. stat. sol. (b) 206, 167 (1998).

[13] O. Di Stefano, S. Savasta, G. Martino, and R. Girlanda, Phys. Rev. B 62, 11071 (2000); Appl. Phys. Lett. 77, 2804 (2000).

[14] O. Di Stefano, S. Savasta, R. Girlanda, J. Appl. Phys. 91, 2302 (2002).

[15] R. F. Schnabel, R. Zimmermann, D. Bimberg, H. Nickel, R. Lösch, and W. Schlapp, Phys. Rev. B 46, 9873 (1992).

[16] e. g. M. O. Scully and M. S. Zubairy, Quantum Optics (Cambridge University Press, Cambridge 1997). 